How to reference this article Iannacito-Provenzano, R. (2019). II plurilinguismo nelle opere teatrali del commediografo molisano Antonio Angelone. Italica Wratislaviensia, 10(1), 281-307.

DOI: http://dx.doi.org/10.15804/IW.2019.10.1.12

Roberta Iannacito-Provenzano

York University, Toronto

roberta@yorku.ca, ORCID: 0000-0002-5205-7178

\title{
IL PLURILINGUISMO NELLE OPERE TEATRALI DEL COMMEDIOGRAFO MOLISANO ANTONIO ANGELONE
}

\section{PLURILINGUALISM IN THE COMEDIES OF MOLISAN PLAYWRIGHT ANTONIO ANGELONE}

\begin{abstract}
This article discusses plurilingualism in the dialect comedies of Molisan playwright Antonio Angelone. After a brief biographical sketch of the author, the rich, expressive, and authentic dialect spoken by his protagonists from the peasant class is analysed. Furthermore, essential details and examples pertaining to Angelone's keen awareness of the challenges of writing in dialect are provided. The most fundamental part of this essay focuses on the plurilingual aspects that - certainly not extraneous to literary production in Italy - fully manifest themselves in Angelone's comedies; this occurs through characters created by a playwright who excels at highlighting how important it is to employ linguistic varieties adapted to the circumstances and needs of the protagonists to make the theatrical experience more authentic and more credible for the reader/viewer. Along with the dialect or dialects, there are examples of Standard Italian, popular Italian, and italiese, or the language of North-American Italians, in the repertoire of the protagonists, pronounced by characters who belong to different generations, who are from different social classes, and who come from different geographical areas.
\end{abstract}

Keywords: literary plurilingualism, dialect plays, dialects, popular Italian, Molisan 


\section{INTRODUZIONE}

Con il presente saggio condivido alcune riflessioni sulle varietà di lingua che si riscontrano nelle commedie dialettali di Antonio Angelone, autore molisano. Nell'articolo "Innovative Transitions: Exploring Italy's Literary Dialects", Hermann Haller osserva: "literary dialects also offer a window to internal and external dialect histories. Language historians will find observations on dialect use and attrition, language shift and language attitudes" (2004, p. 9). Oltre a ciò, continua lo studioso americano: "literary dialect texts themselves yield insights into the form and use of Italian language and dialects through the sociolinguistic study of language variety in literary dialects" (ivi, p. 10) e spiega: "these texts, written down for spoken use during stage performance, with actors and actresses and the theatre public in mind and aimed at total communication, mirror speech situations most closely, despite their differences from ordinary speech such as greater dramatic style or ludic function" (ibidem). Haller avverte comunque: "it must always be remembered of course that we are dealing with literary constructs - letteratura rifless ${ }^{1}$

${ }^{1}$ Croce ha formulato per la prima volta il concetto di letteratura dialettale riflessa distinguendolo appunto dal concetto di letteratura dialettale spontanea o popolare (cf. Croce, 1927); come afferma: "le fiabe e i canti, e gli altri prodotti che i demopsicologi raccolgono dalla tradizione di solito orale e solo di rado scritta, compongono la letteratura propriamente dialettale spontanea o popolare, come anche si chiama, non sottratta già (ché sarebbe impossibile) a ogni e qualsiasi influsso della letteratura colta o nazionale, ma che esprime il costume del volgo [...]; donde il parlare dialettale, nelle conversazioni tra persone del luogo, che si usava e si usa ancora dalle classi colte in alcune regioni d'Italia" (citazione tratta da Beccaria, 1975, p. 21). Per quanto riguarda la letteratura dialettale d'arte o riflessa, spiega Croce che questa "suppone come antecedente e punto di partenza la letteratura nazionale" (ivi, p. 22) e che "il movente effettivo, o il movente principale, della letteratura dialettale riflessa, non che essere l'eversione e la sostituzione della letteratura nazionale, era, per contrario, l'integrazione di questa, la quale le stava dinanzi, non come un nemico, ma come un modello (ibidem). Inoltre, Beccaria, commentando le idee di Croce al riguardo, osserva: "nella letteratura dialettale spontanea il dialetto è adoperato senza la coscienza della dialettalità, come linguaggio nativo che non vuole ne sa distinguersi dalla poesia nazionale in lingua. La letteratura dialettale riflessa invece ha coscienza di essere altra rispetto a quella nazionale" (ivi, p. 20). 
- that are somewhat different from spontaneous oral communication ${ }^{2}$ " (ibidem); afferma dunque che: "literary dialects provide a window for an attempt to reconstruct spoken dialects, thus broadening our knowledge of Italy's linguistic history, and deepening our appreciation of Italy's profoundly plurilingual literary civilization" (ivi, p. 13). Le opere angeloniane offrono quest'opportunità per quanto riguarda il molisano della zona isernina.

\section{ANGELONE E SCRIVERE IN DIALETTO}

Prima di entrare nello specifico, ritengo importante soffermarmi su qualche dettaglio biografico a proposito dell'autore. Dal 1988 ad oggi, Antonio Angelone, nato nel 1933, candidato al premio Nobel per la letteratura (2019), insegnante, noto commediografo, poeta e artista citato da Avolio (2002) nel volume fondamentale sulla dialettologia italiana, I dialetti italiani: storia, struttura e uso, ha pubblicato oltre trenta commedie dialettali, varie raccolte di poesia, sia in lingua che in dialetto, e recentemente ha dato alle stampe un importante glossario dialettale dell'Alto Molise (2015), zona a volte trascurata nelle opere scientifiche. ${ }^{3}$ Della feconda e costante produzione letteraria angeloniana il noto critico Pacitti scrive:

Antonio Angelone, il 'tantillus homo qui tanta fracassa facit' è sempre un magma vulcanico. E spesso mi domando, nel dubbio, se anche per lui la notte 'è fatta per dormire'; se per lui le primavere che si posano sulle spalle con la leggerezza di una farfalla, non debbano consigliare di rallentare il passo

2 Nencioni tratta le nozioni di parlato-recitato e parlato spontaneo nelle opere pirandelliane. Rilevante qui è la seguente osservazione: "è emersa una sapiente mimesi dei fenomeni del parlato; io credo pertanto che ai testi di un tale inventore di parlato teatrale, evidentemente fondato su un'acuta sensibilità, un'attenta osservazione ed una accurata resa dei fenomeni, un linguista possa sicuramente rivolgersi per uno studio del parlato" (1983, p. 178).

3 Esiste, comunque, un vocabolario dialettale di buon livello che riguarda l'intera provincia di Isernia e una parte confinante di quella di Campobasso: Dizionario dialettale. Lessico comparato dei comuni molisani compresi nelle valli interne del Biferno, del Trigno e del Volturno (Bagnoli, Discenza \& Faralli, 2001). 
della marcia, e la danza con le muse, particolarmente con Talia e Erato, le più vezzose. (2014, p. 14)

Angelone vive attualmente a Isernia ed è tuttora impegnato nelle attività culturali, letterarie e artistiche della regione in cui è nato. L'Accademia Lucia Mazzocco Angelone, fondata da lui negli anni 90, ha lo scopo di promuovere artisti, poeti e autori provenienti soprattutto dal Molise ma anche da altre regioni e da altre parti del mondo. Per di più pubblica, quattro volte all'anno, la rivista letteraria Sentieri molisani su cui appaiono articoli che trattano dell'arte, della letteratura e della cultura sia del Molise che a livello più generale.

Nelle sue opere Angelone riproduce fedelmente il dialetto della zona da cui proviene. Questo è chiaro da una breve lettura delle commedie e anche dai numerosi commenti e dalle numerose lodi che ne fanno gli studiosi a proposito. Dialettologi quali Vignuzzi, Avolio, Ruffino e Clivio, per menzionarne qualcuno, hanno contribuito alle opere angeloniane scrivendo introduzioni, prefazioni, postfazioni e brevi commenti critici ai lavori dello scrittore molisano. Commenta Avolio nella prefazione della commedia L'atrajiere, iere e vuojje: "il dialetto, mai come questa volta, ci appare ricco, denso, quasi solare; anche la grafia ampiamente ritoccata e migliorata rispetto alle precedenti prove, si mostra senz'altro più aderente al parlato reale, alla vera pronuncia dei contadini dei villaggi a monte di Isernia" (2005, p. 15). Scrive Clivio, nella postfazione della stessa commedia,

[...] infine come linguista e dialettologo non posso non sottolineare e compiacermi della purezza del parlato che, pur nell'incerta resa grafica, non è inquinato da superflui italianismi come in tanti inutili scritti dialettali che sono piuttosto un italiano storpiato: qui il vernacolo schietto, colto proprio dalle labbra di chi quotidianamente lo parla, rende veri i personaggi tanto che sembra davvero di vederli in carne e ossa, come avverrebbe sul palcoscenico. (2005, p. 67)

Infine, commentando la lingua in Re vuasce sott'alle sctelle (1996), una delle più riuscite e conosciute tra le commedie di Angelone, constata Guido Cecchi: "da questa esperienza positiva e realista deriva l'af- 
fermata preparazione filologica ed estetica della personalità dell'autore che è riuscito a fondere i vari elementi storici, linguistici ed artisticoespressivi nella sua commedia" (2003, p. 45). In effetti, a proposito dello scrivere in dialetto, sarebbe qui importante qualche commento.

Angelone nonostante qualche imprecisione nella resa grafica del dialetto, che tra l'altro corregge ogni volta che scrive una nuova commedia e ne è pienamente cosciente, rende il lavoro di chi esamina le sue opere dal punto di vista linguistico molto interessante perché i suoi scritti rispecchiano, perlopiù esattamente, la parlata locale. Leggendo infatti le opere di Angelone notiamo che l'autore stesso, magari influenzato dai rapporti intimi che riesce a coltivare con noti dialettologi e altri accademici e studiosi, come quelli appena menzionati, ha una presa di coscienza proprio nei confronti della lingua e delle varietà che usa nei suoi scritti e cioè cerca, da una commedia all'altra, di rendere le sue opere più user-friendly, per facilitare la lettura, dedicando in esse più spazio alle avvertenze per quanto riguarda la grafia dialettale e spiegando, per esempio, fenomeni morfologici del dialetto che usa.

Nel 1988, per esempio, Angelone pubblica Re matremoneje, ma è soltanto in una seconda edizione del 2004 che vengono incluse avvertenze e note grammaticali indirizzate al lettore. Nel 1991 dà alle stampe La sperimentazione dei maestri, ma in questa commedia, diversamente da quanto accade in quelle successive, non si riscontrano 'avvertenze' o 'note grammaticali'. Quando nel 1994 scrive, invece, La ruota della fortuna con prefazione di Vignuzzi, include delle avvertenze che spiegano la pronuncia dello schwa e di altri fenomeni morfologici (articoli, aggettivi possessivi, aggettivi dimostrativi) e fornisce accanto alle forme dialettali quelle in lingua. Nel 1995 in Ciccotè offre addirittura un elenco di parole tradotte dal forlivese al dialetto di Capracotta, altro comune dell'Alto Molise e luogo della prima rappresentazione sul palcoscenico di questa commedia. Troviamo esempi quali il forlivese "muacch" che equivale a "pulenda" (it. "polenta') nel capracottese, il forlivese "nzogna o und" equivalente a "und" a Capracotta (it. 'sugna') e, infine, "megnarine" reso con il termine "pesciotta" (it. "pene del maiale') nel capracottese (per un elenco completo di termini si veda Angelone, 1995, p. 13). Si nota dunque che l'autore ritiene importante, per andare dal 
testo scritto in forlivese alla rappresentazione teatrale spostata in un paesino adiacente, usare delle varianti diatopiche e lo fa in modo consapevole per rendere il tutto più autentico. Successivamente, nel 1997 in due commedie, Tra veglie e ssuonne e La vecchia che ne vvuleva murì, non abbiamo più solo avvertenze ma delle brevi note grammaticali ampliate e poi nel 2000 (La banda Centrillo: Brigantaggio postunitario da Vallerotonda a Forli del Sannio) altre correzioni alle brevi note. Tuttavia, è solo nel 2003 e poi nel 2006, come si vede dagli esempi che seguono, che l'autore fornisce, a parte le note di grammatica corrette e rivedute, un giudizio personale a proposito dello scrivere in dialetto.

Vogliamo precisare che alcuni studiosi (dei quali abbiamo il massimo rispetto) nel caso della "e" muta, usano la "e" con dieresi, mentre il digramma $s c$ lo sostituiscono con una $s[\ldots]$ che mentre potrebbero essere giustificati dal punto di vista scientifico, renderebbero d'altra parte, talmente artificiosa la scrittura da esserne poi quasi impossibile la lettura. (Angelone, 2003, pp. 6-7)

Lo stesso accade nel 2006 con la stesura di Felemena la lengacciuta e Alfonz re cengenare. Alle brevi note grammaticali l'autore aggiunge delle osservazioni a proposito del gruppo nominale e delle desinenze, dei commenti sui verbi ausiliari e sulla particella ne ecc. Inoltre, spiega Angelone: "siamo pienamente d'accordo con gli studiosi di dialettologia che la scrittura del dialetto è talmente difficile, da creare qualche difficoltà anche agli specialisti della materia. Perciò non voglia meravigliarsi il lettore se trova qualche discordanza nella lettura tra una commedia e l'altra" (2006, p. 19). Questi brevi cenni indicano la risolutezza dell'autore per quanto riguarda la cura delle lingue e delle forme che vengono da lui impiegate nelle commedie dialettali.

È importante ricordare che Angelone per tutta la vita è stato insegnante nelle scuole di Forli del Sannio, una frazione di Vastogirardi nell'Alto Molise, e nei comuni contigui. A parte la conoscenza dell'autore del dialetto e della lingua italiana che quotidianamente impartiva ai suoi alunni, come spiega Martelli: “[...] ed insieme profondamente e solidali integrati nella realtà sociale contadina in cui vivono, i maestri di scuola incarnano un ruolo di cerniera tra la cultura popolare e quella 
dotta" (1996a, p. 302). Di una delle sue opere più riuscite, Ciccotè, in cui Angelone tratta il ciclo del maiale e ciò che questo rito significava per i paesani, spiega Iemma: "ad Angelone non occorreva, quando componeva Ciccotè, come nei migliori studi demologici, operare ricerche sul campo: lui nel campo popolare c'era già, gli bastava solo saper raccogliere i frutti ed in questo si è dimostrato maestro perché l'intera situazione demologica nella quale muove i suoi personaggi non gli è estranea" (1997, p. 83).

Attraverso protagonisti che usano un dialetto espressivo e autentico, tratti dal mondo contadino a cui appartenevano anche lui e i suoi genitori, il nostro commediografo documenta in modo meticoloso, per esempio, la terminologia dialettale relativa a diversi riti e cicli annuali in pericolo d'estinzione (cf. Iannacito-Provenzano, 2009, p. 90). Per Paone, noto esperto di cultura molisana e autore di numerosi libri sulla transumanza in quest'area e in zone adiacenti, "il dialetto rappresenta il passaggio della comunicazione dal disegno della parola scritta, la sua presenza nell'evoluzione dell'uomo assume il significato della scoperta del fuoco e, con tutti i limiti, rimane la forma più immediata, diretta e fedele di trasmissione dei sentimenti più autentici della persona [...]" (2006, p. 11). Tutto ciò è pienamente visibile nelle opere angeloniane. Infatti, il dialetto - "ultima frontiera della libertà e dell'autenticità espressiva" (Folena, 1991, p. 122), posto sulla bocca di diversi personaggi nelle commedie studiate - viene usato, "al polo opposto dell'espressione ricercata e artificiale e insicura della lingua egemone" (ivi, p. 125), per commentare e anche criticare non solo momenti storici ( $v$. infra), ma anche argomenti e problemi contemporanei. Nelle opere angeloniane, il personaggio, dotato di saggezza popolare, che commenta temi moderni molto spesso di natura politica o sociale e rimpiange i tempi passati, è Nonna Teresa in Re vuasce sott'alle sctelle. L'autore spinge i limiti e usa il dialetto pronunciato dalla vecchietta per trattare argomenti quali l'inquinamento e Tangentopoli dimostrando, come osserva Martelli nella prefazione, che:

[...] al dialetto non sono precluse le strade del presente che anche col dialetto e con il bagaglio dei suoi valori, esperienze e sapori si può affrontare il pre- 
sente. Anche il grande tema della società contemporanea, quello ecologico, può trovare posto in una rappresentazione dialettale, soprattutto nelle mani di Angelone, che ne fa occasione ancora una volta per una garbata ed ironica contrapposizione tra passato e presente, tra civiltà contadina e civiltà moderna. (1996 b, p. 8)

Tutto ciò si rifà a Haller che spiega:

[...] the dialect [became] synonymous with the language of reason and common sense. Previously used as the register of laughter and equivalent with the comical, the dialect began gradually to gain a wider spectrum of uses of social specification, and the playwrights tended to identify with them and their characters rather than with the artificial speech forms. (1999, p. 43)

Queste parole sono echeggiate da De Martino Cappuccio che scrive:

[...] far from being folkloristic, contemporary dialect theatre has acted more and more as the goad of power, using vernacular as a particularly effective medium to reach the people's hearts and to raise social and political awareness. Indeed, Eduardo De Filippo used Neapolitan dialect to address socially relevant issues such as prostitution, mafia, and social justice, whereas Dario Fo employed Milanese, Bergamask, and Paduan dialects and the hotchpotch (sic) of the grammelot in political theatre. $(2012$, p. 81)

\section{OLTRE IL DIALETTO}

Nella mia breve indagine che tratta soltanto alcune commedie dell'intero corpus di Angelone, sono stati reperiti esempi che servono da spie linguistiche che mostrano l'uso che l'autore fa di altre varietà linguistiche, accanto al dialetto, usate in diverse situazioni. Angelone, come notato sopra, non è un autore dilettante che scrive per nostalgia. Data la sua formazione, conosce bene l'italiano, gli scrittori latini e i grandi autori della tradizione letteraria italiana. Per fare un breve esempio, in Voci d'oltretomba: Da Micheleccia a Colle Pertino (2007), commentata da Giorgio Barberi Squarotti (2009) in una comunicazione personale all'autore, Angelone presenta parte in prosa e parte in dialogo, parte in lingua e parte in dialetto degli intrecci in cui appaiono Seneca, Orazio, Dante ecc. Non è il caso qui di entrare nei dettagli perché quest'opera meriterebbe una 
ricerca più approfondita a parte. Comunque, constata Barberi Squarotti: "Voci d'oltretomba è un'opera singolare, che ridà voce e futuro ai personaggi autentici suasivi del mondo perduto e fa riapparire i morti in forza della riconquista del sacro contro la dissoluzione e la verità del nostro tempo" (2009, p. 187). Tutto questo ci ricorda tra l'altro, per esempio, La Veneixiana (opera citata da Trifone, 2000, p. 60) dove l'anonimo autore mette in bocca ai suoi personaggi la lingua che parlerebbero nella realtà e ci fa pensare al 'plurilinguismo organico', termine usato da Folena (1991, p. xi). Commentato da vari studiosi (Folena, 1991; Marcato, 2012; Trifone, 2000; Paccagnella, 1984), sappiamo che il plurilinguismo non è certamente estraneo alla produzione letteraria in Italia soprattutto per quanto riguarda il teatro dialettale. Come spiega Haller: "plurilingualism may be considered as one of the dominant features of dialect plays, from Ruzante's comedies to Dario Fo's invented orgiastic pastiche [...] and it appeared in a variety of forms, from the coexistence of different languages or dialects in the same text to that of different stylistic registers of the same language" $(1999$, p. 50), parole echeggiate da Trifone (2000, p. 59) e da Carlson (2006, p. 68) quando parlano del cosiddetto "plurilinguismo orizzontale" in opposizione al concetto di "plurilinguismo verticale" (ibidem). Specificamente a proposito del tema del plurilinguismo teatrale, spiega Folena che esso "si presenta a storici e studiosi di lingua e di teatro come problema storiografico non marginale, quello della 'questione della lingua' al livello teatrale [...]" (1983, p.1489).

Basta qualche breve esempio per illustrare questi aspetti plurilinguistici nelle opere di Angelone. Si notano, prima di tutto, scelte sociolinguistiche e più precisamente diastratiche che mostrano l'oscillazione tra dialetto e altre varietà. Nella commedia La sperimentazione dei maestri (1991) è evidente soprattutto l'uso del dialetto sulla bocca degli anziani che interrogano i ragazzi per vedere che cosa imparano a scuola, come nella scena che segue. Gli alunni, invece, usano soltanto l'italiano. Le seguenti battute serviranno ad illustrare questo ${ }^{4}$.

4 In quest'articolo non viene usata la trascrizione fonetica (IPA) per le citazioni in dialetto (tranne in un istante a p. 16). Si è preferito invece citare le commedie dialettali senza modificazioni. Pertanto si segnala che di solito la /e/ non accentata ha il suono 
1. Zia Carmela: Me sapiss dice com ze chiama chella carruzzella che porta ngoppa ndutt alla mundagna, andò viann a scià?

(it. Sapresti dirmi come si chiama quella seggiovia che ci porta sopra la montagna, dove si va a sciare?)

Rugiadino: Quella non è una carrozzella, si chiama seggiovia.

Zia Carmela: Te piacene r'ariange?

(it. Ti piacciono le arance?)

Rugiadino: Come, se non mi piacciono!

Zia Carmela: E me sià dice dandò vienn chisct ariange che ze magnamm nu?

(it. Sai dirmi da dove vengono queste arance che (si) mangiamo (noi)?)

Rugiadino: Queste arance vengono quasi tutte dalla Sicilia e dalla Calabria. Poche dalla zona di Napoli.

(Angelone, 1991, p. 34).

Angelone fa ciò per dimostrare che i maestri - a differenza di ciò che pensava la gente del paese, che spesso raccontava pettegolezzi - non facevano re muacche dentre a re stanzine (1991, p. 18), cioè 'la polenta nello stanzino', ma svolgevano realmente il loro lavoro in aula, e lo facevano bene. Angelone mette l'italiano sulla bocca dei ragazzi precisamente per valorizzare il lavoro dei maestri, essendo stato maestro anche lui. Ne La tagliola (2013b) si riscontra un altro esempio di giovani che, diversamente dagli esempi appena presentati, usano il dialetto per correggere gli anziani.

2. Fiorella: Prima di tutt, care tata, te lo songh ditt mill vote ca ne nze dice 'carboniere' ma ze dice ca-ra-bi-nieri. Quand re chiam accusci la ggend ze mett a rride.

(it. Prima di tutto caro papà, te l'ho detto mille volte che non si dice 'carboniere' ma si dice ca-ra-bi-nieri. Quando li chiami così, la gente si mette a ridere.)

schwa /a/, ovvero muto in questa zona dialettale. A volte il commediografo lo trascura completamente specialmente in posizione finale oppure lo trascrive con /e/. 
Mechele (alla figlia): Quanda ne veia truvann pure tu mo, o re chiame carbinieri come dice tu ca ze chiamane, o carboniere come re chiami', semb carboniere so.

(it. Quante storie! O li chiami 'carabinieri' come dici tu che si chiamano o 'carboniere' come li chiamo io, sempre 'carboniere' sono.)

(Angelone, 2013b, p. 22).

Spiega Troiano a questo proposito:

posti sulla bocca dei personaggi più anziani, o non istruiti, i malapropismi, gli equivoci verbali, le storpiature, sono stati sempre una risorsa di comicità sulla scena per lo scrittore di commedie o di farse popolari, come dimostra in minima parte, questo scambio di battute che vuole essere prima di tutto un contrasto, tra padre e figlia, di mentalità, atteggiamenti, comportamenti linguistici. (2013. p. 7)

Benché si noti il valore comico della battuta, dobbiamo ricordare, come quanto detto sopra, che anche qui le scelte linguistiche vengono fatte di proposito da un commediografo pienamente conscio del repertorio linguistico dei personaggi da lui creati. A volte i personaggi stessi pronunciano frasi che indicano che anche loro si rendono conto di parlare solo dialetto, mentre gli altri personaggi con cui interagiscono usano altre varietà. Per esempio, in Fuga in Paradiso (2013a) il commediografo, per ambientare l'azione, sceglie il Monte Athos, territorio autonomo della Grecia dove non sono ammesse né donne né bestie di sesso femminile. A differenza di alcune commedie precedenti, l'autore va oltre la vita in paese e sposta letteralmente i suoi personaggi, tra l'altro uomini e donne che riescono a infilarsi nel territorio nonostante le leggi glielo proibiscano, da un ambiente più o meno sicuro, o meglio conosciuto, a uno completamente diverso per far sì che si rendano conto dei problemi altrui che sono, essenzialmente, preoccupazioni universali. Durante il percorso, i nove pellegrini che parlano solo in dialetto tra di loro, incontrano diversi personaggi non-molisani quali il Guardiano, la Dea Astrea e San Giovanni. Questi ultimi parlano solo italiano sia tra di loro che con i pellegrini.

Commentando la Commedia dell'Arte, la società milanese e la mentalità della borghesia, scrive Trifone: "il plurilinguismo diviene così un sensibile indicatore delle divisioni e delle tensioni presenti all'interno 
della società" (2000, p. 61), parole echeggiate da de Martino Cappuccio: "theatre in 'italiano letterario' represented the bourgeoisie, whereas the masses spoke through dialect theatre, which, albeit linguistically limited, portrayed the life of large sections of the population" (2012, p. 79). È evidente che, come si nota qui di seguito, Angelone rende chiarissima la distinzione di classe tra i due gruppi (anche se alcuni di questi sono figure religiose, fatto che aprirebbe tutt'un altro discorso) e dimostra quanto in effetti siano coscienti i protagonisti molisani di questa situazione linguistica quando fa pronunciare le seguenti parole ad Isabella alla Dea Astrea che parla solo italiano.

3. Isabella: Signora, io non sò parlare taliano perché non sono studiato, so parlare soltand 'ndialett. Nesciune de nu sà parlà taliane. Nè ssacce chi sete. (it. Isabella: Signora, io non so parlare italiano perché non ho studiato, so parlare soltanto il dialetto. Nessuno di noi sa parlare italiano. Non so chi siete.)

Astrea: Chiamami Astrea e non preoccuparti di darmi degli attributi o sapere chi sono. Va bene, ho capito, parla come vuoi, basta che mi dici perché e come ti trovi in questo luogo.

Isabella: Signora Astrea, le sacce, pe ssendute dice, ca ji come femmen 'nge puteva menì a re Mond Athoss, però da nu ze dice la necessità romb la legge. Re cuvern a nnu z'a miss 'mmiesa 'nna viia.

(it. Isabella: Signora Astrea, lo so, per sentito dire, che io come donna non sarei potuta venire al Monte Athos, però da noi si dice che la necessità rompe la legge. Il governo ci ha messo per la strada.)

(Angelone, 2013a, pp. 24-25).

Sarebbe importante notare in questo scambio l'italiano popolare, cioè "quel modo di esprimersi di un incolto che, sotta la spinta di comunicare e senza addestramento, maneggia quella che ottimisticamente si chiama la lingua 'nazionale', l'italiano" (De Mauro, 1970, p. 49). Questa varietà, caratterizzata da vari studiosi con sfumature leggermente diverse $^{5}$, è pienamente visibile nelle commedie dialettali di Angelone

${ }^{5}$ Cf. a titolo d'esempio: Cortelazzo, 1972; Sabatini, 1985; Berruto, 1987; D’Achille, 1994. 
come dimostra l'esempio precedente e come vedremo qui di seguito. Nello scambio precedente si notano esempi di code-mixing, o enunciati mistilingui, riscontrabili in quasi tutte le commedie di Angelone. In questo caso abbiamo l'oscillazione tra 'so', 'sò' e 'sacce' per la prima persona singolare del verbo "sapé" (it. sapere). Inoltre, nella stessa frase è interessante l'uso di 'perché' e 'necessità', termini scritti in italiano, ma probabilmente pronunciati diversamente, cioè in dialetto, sul palcoscenico. Per quanto riguarda il termine 'soltando' (it. soltanto), non comune nei dialetti dell'Alto Molise, viene usato accanto alla parola più comune 'suole'. Qui viene usato 'soltand' con la consonante sonora /d/ in posizione post-nasale tipica di questa zona e senza vocale finale che sarebbe, comunque, schwa. Altro esempio di questo si riscontra in L'atrajiere, iere e vuojje.

4. Meruccia: Suole re Pataterne ce po' penza. Suole quire e nnesciun'uatere cchiù.

Annarella (risponde poi): Ma ne nnada dice però ca vu sete cambata soltand che re sedure vuosctre.

(it. Meruccia: Solo il Padre eterno ci può pensare. Solo quello e nessun altro più. Annarella (risponde poi): Ma non devi dire che voi avete campato solo grazie ai vostri sacrifici. / letteralmente 'ai vostri sudori')

(Angelone, 2005, p. 40)

Ancora a proposito dell'italiano popolare, se ne riscontrano numerosi esempi nell'opera La banda Centrillo: Brigantaggio postunitario da Vallerotonda a Forli del Sannio. Scritta nel 2000, in questa commedia Angelone tratta il brigantaggio in Molise e ci riporta verso gli anni attorno al 1860. L'opera è il risultato di quattro anni di ricerche da parte dell'autore negli archivi di Stato di Isernia, Campobasso e Caserta in cui ha esaminato gli atti processuali relativi ai briganti e alle loro attività nelle zone dell'Alto Molise. Quello che ci insegna la commedia riguardo alla storia di questo periodo e del brigantaggio è, senza dubbi, di alto valore perché - come sempre - Angelone documenta nei più minuziosi dettagli la costituzione della banda Centrillo, gli atti di giuramento di fedeltà a tale banda, le attività da svolgere, la descrizione dei luoghi d'incontro dei briganti, che cosa facevano quando invadevano un villag- 
gio ecc. All'inizio della commedia Angelone fornisce anche un elenco dei personaggi e mette accanto ai nomi il comune di provenienza di ognuno dei protagonisti (per esempio "Domenico Coia, detto Centrillo di Castelnuovo"; "Mariano Centracchio di Rionero Sannitico"; "Nicola Onorato di Forlì del Sannio"; "Pasquale Berardi di Acquaviva d'Isernia"; 2000, p. 13).

La caratteristica, comunque, che più colpisce il lettore è l'uso di un tipo di pastiche linguistico tra dialetto, ovvero dialetti di alcune zone, italiano e italiano popolare. Angelone, a parte la buona conoscenza dei dialetti delle zone adiacenti a Forli del Sannio, usa gli atti processuali tratti da Forli del Sannio, ma anche da vari comuni, come Acquaviva d'Isernia, Fornelli, Vallerotonda (comune che non si trova nel Molise, ma nel Lazio, in provincia di Frosinone; fino al 1927 ha fatto parte della provincia campana di Caserta), Castelnuovo (annesso a Scapoli in passato, ma oggi chiamato Castelnuovo al Volturno, frazione di Rocchetta al Volturno), Montenero Val Cocchiara e Roccasicura per riportare fedelmente la lingua usata dai briganti dell'epoca. Come spiega Avolio nella prefazione, Angelone:

[...] dà vita ad un originale impasto di "italiano" e dialetto anzi dialetti, perché nelle battute sono pur sempre riconoscibili le diverse varianti municipali della zona, messe in bocca ai singoli personaggi, di cui Angelone ha accertato l'esatto luogo di origine, impasto che, in più punti, ricorda da vicino proprio quello dei documenti d'archivio, delle risposte fornite dai briganti durante gli interrogatori e i processi - e verbalizzate dai loro inquisitori - o dei loro stessi componimenti, non di rado autobiografici (insegnativi, a questo proposito, gli studi compiuti qualche anno fa da Nicola De Blasi sulla lingua del brigante Gè). (2000, p. 9)

Nella commedia troviamo Centrillo, il capo-brigante che usa perlopiù il proprio dialetto, ma che spesso, in situazioni diverse, cerca di parlare 'italiano' e dunque sono evidenti anche qui molti casi di codemixing. Fin dal primo atto quando parla al pubblico a proposito della costituzione della banda e delle attività da svolgere troviamo: 
5. Centrillo: (Girandosi intorno e parlando ad alta voce) Soltand chescta ${ }^{6}$ ce pò penzà a ffà giusctizeia; soltand chescta che port ngoppa 'lla spalla! E tè pure re taglie buone. Ogne botta na coccia nderra. Chescta setuazione ne mbò propria i. Ne vvà e ne vvà. Che Vettoreie Emanuele la vita ne nnè vita. (it. Centrillo: (Girandosi intorno e parlando ad alta voce) Soltanto questa ci può pensare a fare giustizia; soltanto questa che porto sulla spalla. Ė anche ben affilata. Ogni colpo, una testa per terra. Questa situazione non può più andare avanti. Non va e non va. Con Vittorio Emanuele, la vita non è vita.) (Angelone, 2000, p. 18)

In questa prima parte del suo intervento vediamo più o meno un dialetto autentico pur con l'eccezione di qualche esempio verso l'italiano. Usa anche lui 'soltand' come Isabella nell'esempio 3. Nonostante ciò, nel lungo discorso di Centrillo la tendenza a italianizzare è pienamente visibile e si riscontra, negli esempi che seguono, una serie di codemixing.

6. Centrillo: Mo le seme propreie passate le guaie e pure assaie e no prima. Li garibaldini devone sparire da le Regne de Napule, ze ne devene andare e pure subbete, per Dio. Tutti li signori ruffiani di Garibaldi, li co-si-dde-tti garibaldini pure lore devene scomparì non sole da Castelnuovo ma da tutt le Regne de Napule. Li comand deve returnà a Frangesch Second a tutti li costi se nnò part li cuoglie a tutti: garibaldini e liberali, uomini e femmene, vecchi e bambini. Cari liberalacci, arrauniteve attorno a noi se non volet'ess massacrati. Abbasso Garibaldi e li garibaldini, abbasso li particolari, abbasso Vittorio Emanuele. (Angelone, 2000, p.18)

(it. Centrillo: Ora, e non prima, abbiamo proprio passato dei guai e anche molti. I garibaldini devono sparire dal Regno di Napoli, se ne devono andare e pure subito, per Dio. Tutti i signori ruffiani di Garibaldi, i cosiddetti garibaldini pure loro devono scomparire non solo da Castelnuovo ma da tutto il Regno di Napoli. Il comando deve tornare a Francesco II a tutti i costi sennò togliamo la testa a tutti: garibaldini e liberali, uomini e donne, vecchi e bambini. Cari liberalacci, mettetevi con noi se non volete essere massacrati. Abbasso Garibaldi e i garibaldini, abbasso i particolari, abbasso Vittorio Emanuele.)

${ }^{6}$ Si riferisce a una grande roncola con un lungo manico che porta sulla spalla. 
Tra gli esempi notevoli nel suo discorso abbiamo: 'le guaie' (it. i guai), che sembra essere un esempio di metaplasmo, con parziale italianizzazione fonetica e, forse, ma dipende dal tipo di dialetto, morfologica, di genere, che nei dialetti di questa zona sarebbe maschile e probabilmente reso, in molti dialetti della provincia d'Isernia con il termine / uaja/ come a Castelnuovo e a Forli del Sannio (IS), spesso preceduto da 'tanda' o 'quanda' (e.g. tanda uajo, it. tanti guai), entrambi confermati da Angelone stesso, e riscontrabili anche nell'opera di Santilli, Isernia e il suo dialetto, dove abbiamo voci quali 'uoje/vuoje' e 'uaje/vuaje' per guaio (1988, p. 34) ${ }^{7}$; a Roccasicura (IS) e a Villa San Michele, frazione di Vastogirardi (IS) si trovano, rispettivamene $/ \mathrm{r}$ vèa $/{ }^{8} \mathrm{e} / \mathrm{r}$ ə vjà/ tutti e due con $/ \mathrm{v}-{ }^{9}$ iniziale per $i$ guai. Nell'esempio 6 c'è anche l'uso dell'ausiliare senza troncamento finale ('sparire' dove ci si aspetterebbe 'sparì') accanto alla forma con apocope ('scomparì'); l'uso del verbo simile alla forma italiana 'dovere' ('devene', 'deve', ecc.), ma sappiamo che per rendere il significato di 'dovere' in questa zona si usano spesso forme quali 'adda', 'ada' ${ }^{10}$, come visto sopra pronunciato da Meruccia (e.g. 'adda/ada fa', it. devi/deve fare). Si notano anche delle differenze nell'ortografia del nome Vittorio Emanuele.

Interessanti sono anche le forme del pronome dimostrativo. Si nota prima di tutto l'oscillazione tra 'queshto/a' e 'questo/a' (it. 'questo/a'). Sia la forma tipica di questa zona dell'Alto Molise, con la consonante

7 Voci simili si riscontrano anche nella provincia di Campobasso e dunque abbiamo, per esempio, a Ripalimosani '(g)uaie' (singolare) e '(g)uieie' (plurale) (Minadeo, 1996, p. 57); a Bonefro, "'u guaje" e "u'uaje" (Colabella, 1993, p. 154).

${ }^{8}$ Cf. Rohlfs, 1966, §298, per una spiegazione della palatalizzazione o della velarizzazione entrambe provocate dall'articolo determinativo in esempi come "ro keanə" (it. i cani) o "l'easənə" (it. gli asini) appunto a Roccasicura che oggi fa parte della provincia d'Isernia.

9 Cf. Rohlfs, 1966, §§155, 167 e 168, per altri esempi pertinenti a /g-/, /v-/, /w-/.

${ }^{10}$ Come segnalato anche da Santilli (1988) per il dialetto d'Isernia però con rotacismo tipico di questa zona: avé + ra + infinito (avere $+\mathrm{da}+$ infinito) come per esempio in "tu ara i" (it. tu devi andare) mentre nella prima persona sarebbe "i aja i" (it. io devo andare) senza rotacismo (v. Santilli, 1988, pp.73-74; cf. anche Iannacito, 2006, pp.157-160). 
fricativa postalveolare $/ \check{\mathbf{s}} /{ }^{11}$ prima della consonante occlusiva sorda $/ \mathrm{t} /$ (si nota anche nella pronuncia di Casctelnuovo qui di seguito), che l'altra all'italiana con la consonante fricativa alveolare /s/ preposta alla / $/$, vengono impiegate da Centrillo quando parla con i membri della sua banda. Inoltre, Centrillo usa spesso 'chescta' (it. questa) come nell'esempio 5. L'esempio che segue dimostra l'uso di 'chescta' accanto all'uso di 'quescta'.

7. Centrillo: (Prende diversi paletti, prende la pietra, vi scrive sopra il nome di alcuni paesi, le dispone in modo irregolare a terra e le collega con i paletti. Poi si rivolge ai compagni.) Sctetema 'ssendì. Chescta preta chiù grossa rappresenda la grotta; chesct'ata Casctelnuovo, quescta Volturn, quescta Cardite, chescta Schapule, chescta Cerr al Volturn, quescta Casctel San Vengenz, quescta Colì, quescta l'Accuvive, quescta Riunero e quescta Forli. D'accord? (Angelone, 2000, p. 25)

(it. Centrillo: (Prende diversi paletti, prende la pietra, vi scrive sopra il nome di alcuni paesi, le dispone in modo irregolare a terra e le collega con i paletti. Poi si rivolge ai compagni.) Ascoltatemi! Questa pietra più grande rappresenta la grotta; quest'altra Castelnuovo, questa Volturno, questa Cardito, questa Scapoli, questa Cerro al Volturno, questa Castel San Vincenzo, questa Colli al Volturno, questa Acquaviva d'Isernia, questa Rionero Sannitico e questa Forli del Sannio. D'accordo?)

Altro caso interessante di code-mixing, dove si notano tentativi verso 1'italianizzazione, si trova nel discorso di Caterina, la nuora di Antonio Fisco, proprietario di una delle case che vengono assalite dai briganti a Forli del Sannio. Mentre sta dando da mangiare al figlio, entrano i briganti.

8. Caterina: (Spaventata si mette a gridare piangendo disperatamente.) Ma iio che ti sono fatte de male! Iio sone na povera desgrazeiata; iio ne nzone fatt male maie a nisciuno. Che cosa siame fatt de male iio e quescta povera anema di Diia. (Angelone, 2000, p.33)

(it. Caterina: (Spaventata si mette a gridare piangendo disperatamente.) Ma io che ho fatto di male. Sono una povera disgraziata; non ho fatto mai male a nessuno. Che cosa abbiamo fatto di male io e questa povera anima di Dio.)

${ }^{11}$ Cf. Rohlfs, 1966, §266. 
Nel dialetto di Forli del Sannio in tutti i tempi composti del passato si usa il verbo ausiliare 'esse' (it. essere) per i verbi transitivi e intransitivi tranne nella terza persona singolare dove si usa 'avé' (it. avere). ${ }^{12}$ Nel discorso sopraccitato, Caterina usa forme quali 'sone', 'nzone fatte' e 'siame fatt'. Nel primo esempio vediamo i tentativi di Caterina verso l'italianizzazione perché la forma dialettale sarebbe 'songhə' che si riscontra parecchie volte nelle commedie di Angelone e che si trova anche, per esempio, in molti altri dialetti dell'Alto Molise e della provincia d'Isernia in generale. ${ }^{13}$ Nei due esempi al passato, l'ausiliare è quello che ci si aspetterebbe però anche in queste forme, è italianizzato. Infatti, nell'esempio 'siame fatt', l'ausiliare nel forlivese, come in quello delle zone adiacenti, sarebbe 'semmə'. Un esempio simile si trova nel terzo atto mentre Centrillo parla con Francesco:

9. Centrillo: Quest è l'elengh de tutt quell che abbiame prese; consegnatele a Vettoreie Emanuele. Lui ve darà li dinari pe recumbrà tutt quell che vi siame prese. (Angelone, 2000, pp. 57-58)

(it. Centrillo: Questo è l'elenco di tutto quello che abbiamo preso; consegnatelo a Vittorio Emanuele. Lui vi darà i denari per riacquistare tutto quello che abbiamo preso.)

Addirittura qui Centrillo usa, nella prima frase, il verbo 'avere', come in italiano, e poi 'essere' nella seconda proprio come lo usa Caterina in 'siame fatte' citato nell' esempio 8. Da notare qui, a differenza dell'esempio 7, sarebbe la forma italianizzata del dimostrativo senza $/ \check{s} /$.

Per ritornare a Caterina, quando gli stessi briganti le vogliono rubare gli orecchini e la fede, vediamo lo stesso fenomeno:

10. Purtateve li orecchine ma restituiteme la fede che me dett quel povere marite miia. (Angelone, 2000, p.34)

(it. Portatevi gli orecchini ma restituitemi la fede che mi diede quel povero marito mio.)

12 È un fenomeno che si nota dalle commedie dialettali di Angelone e anche dalla mia ricerca sul campo (cf. anche Iannacito, 2005).

${ }_{13}$ Cf. Rohlfs, 1966, §540; Santilli, 1988, p. 68; Iannacito, 2006, p.154. 
Anche questi esempi dimostrano che Caterina tenta di usare meno dialetto e più italiano con gli invasori. Notevole è l'uso del verbo 'restituire' perché in questi dialetti sarebbe più comune usare forme quali 'ardéteme' o 'ardàteme', 'arrennéteme' (it. ridatemi). Quando si rende conto che le suppliche sono inutili, Caterina va su tutte le furie e usa il dialetto in modo espressivo e autentico. Si nota l'uso del dimostrativo maschile singolare 'quel' sopra, mentre in ciò che segue si trova 'quire' la forma dialettale con il significato di quello in queste zone dell'Alto Molise. Oltre a ciò, si riscontrano, in quello che segue, alcuni fenomeni caratterizzanti i dialetti di questa zona, e cioè la metafonesi anche $\mathrm{su} / \mathrm{a} /$ tonica (sctià, $i t$. stai) ${ }^{14}$, il dimostrativo neutro non metafonetico chesct 'questo, ciò', che si oppone al maschile metafonetico 'quire' (cf. Iannacito, 2006, p. 139), rispetto al quale perde anche il nesso labiovelare, oltre al più diffuso costrutto 'a cornice' "Z'a miss mmiesa nna viia z'a miss", 'ci ha messi in mezzo a una strada, ci ha messi!', che si ritrova anche nelle varietà di italiano regionale.

11. Caterina: (Si getta a terra gridando e tirandosi i capelli.) Mamma e ch'è ssucciess massera ch'è ssucciess! Sandandoneie miia aiutame tu. Ch'è ssucciess massera denda 'scta casa meia! Amodiio miia andonna sctià massera, andonna sctià, Che me si fatt massera, Amodiio, che me si fatt. Ndenive propia cchiù tiemb de ii 'lla candina, nde nive propia chiiù tiemb! Diia miia che d'è chesct, che d'è chesct Madonna meia aiutame tu, aiutame te, Madonna, che vvuleva Vettoreie Emanuele, che vvuleva. Z'a miss mmiesa nna viia z'a miss. Che ze n'aveva fà quire marite miia de metteze ngh re Garebaldine, che ze n'aveva fa. (Angelone, 2000, p. 34)

(it. Caterina: (Si getta a terra gridando e tirandosi i capelli.) Mamma, che cosa è successo stasera, che cosa è successo! Sant'Antonio mio aiutami tu! Che cosa è successo stasera in casa mia! Amodio mio dove sei stasera, dove sei? Che cosa mi ha fatto stasera, Amodio, che cosa. Proprio stasera sei dovuto andare alla cantina! Dio mio che cosa è questo, che cosa è questo Madonna mia aiutami tu, aiutami tu Madonna, che voleva Vittorio Emanuele, che voleva! Ci ha messo per strada! Che cosa interessava a mio marito mettersi con i garibaldini?)

14 Iannacito tratta la metafonesi della /a/ tonica nel dialetto di Villa San Michele (IS), frazione a pochi chilometri da Forli del Sannio (2006, pp. 48-70); si veda la bibliografia ivi riferita, tra cui p. es. Piemontese, 1993, p. 13. 
In questa e nelle altre opere di Angelone, l'oscillazione tra la scelta dei verbi ausiliari (p. es. Centrillo: "me meraviglie che non haie sapute"; "ne le si sapute"; Angelone, 2000, p. 21), 1'oscillazione nelle forme dei verbi della prima persona plurale (p. es. perdiame, andiame, portiame, partiame, dobbiame vs partimme, firmamme) e l'oscillazione che si riscontra nel sistema pronominale (p. es. riferendosi al patto: "le firmamme subete" (it. lo firmammo subito; ibidem) vs "come re facette" (it. come lo fece; ivi, p. 25) vs "re ${ }^{15}$ canussce" (Centrillo che parla al Sindaco; ivi, p. 31), "te facce sicche" vs "ti facce secco" (it. ti faccio fuori; Centrillo al Sindaco; ivi, pp. 31-32)) meriterebbero una ricerca approfondita, ma per ragioni di brevità non è il caso di entrare nei dettagli. Vale lo stesso per la frase ipotetica nelle opere angeloniane, struttura molto interessante e complessa trattata già ampiamente in un'altra sede (cf. Iannacito, 2005, pp. 498-519). Mi limito soltanto a qualche esempio illustrativo e rilevante nell'ambito delle varietà e delle oscillazioni nei repertori linguistici individuali di alcuni personaggi. Per esempio, quando parla Nonna Teresa in Re vuasce sotta'lle sctelle si notano due possibilità diverse per esprimere l'irrealtà nel presente: la prima con l'imperfetto del congiuntivo nelle due clausole, tipica di questa zona, e l'altra simile alla costruzione italiana, ma anch'essa dialettale, con il condizionale nell'apodosi (cf. AIS 1633, 1634, punto 666, Roccasicura)

Se + imperfetto del congiuntivo + imperfetto del congiuntivo

12. Nonna Teresa: Se ii putéss turnà rrète ce turnasse da mo che tutt ch'eva puverella. (Angelone, 1996, p. 25)

(it. Se potessi tornare in dietro, ci tornerei da ora anche se ero povera.)

Se + imperfetto del congiuntivo + condizionale presente

13. Nonna Teresa: Se ce putéssa'reiì rrète da mo c' iareièra ${ }^{16}$ pure s' avéssa purtà le pèzz ngure (ivi, p. 48).

15 L'oscillazione tra re e le potrebbe in parte sottendere la distinzione, ancora piuttosto vitale nel dialetto, tra articolo maschile (re) e neutro (le); cf. AIS 19 (per il maschile), 1340 (per il neutro) nel punto 666, Roccasicura, e Rohlfs, 1966, § 419; §420.

${ }_{16}$ Qui il condizionale è quello di tipo arcaico, derivante dal piuccheperfetto latino (cf. Rohlfs, 1966, §751) e diverso da quello in -ia che compare nell'esempio 15 (sarria 'sarei') (ivi, §745, cf. Bagnoli, Discenza \& Faralli, 2001, p. 30; Iannacito, 2005, pp. 503-507, 2006, pp. 206-207). 
(it. Se ci potessi ritornare, ci andrei da ora pure se dovessi portare i pantaloni rattoppati.)

Per esprimere l'irrealtà nel passato, nel repertorio di Capedefierre in Tra veglie e ssuonne si trovano esempi con il trapassato del congiuntivo sia in protasi che in apodosi come nella prima frase, mentre nella seconda si nota l'imperfetto dell'indicativo nella subordinata e il condizionale passato nella clausola principale.

$\mathrm{Se}+$ trapassato del congiuntivo + trapassato del congiuntivo

14. Capedefierre: Se fuss sctate per re figlie de Ndunietta ne me fuss tuote mangh gnend. (Angelone, 1997, p. 31)

(it. Se fosse stato per il figlio di Ndunietta (Antonietta), non mi sarei preso nemmeno niente.)

$\mathrm{Se}+$ imperfetto dell'indicativo + condizionale passato

15. Capedefierre: $[\ldots]$ se la capenera ne me ze tuglieva tutt chell lena pe na vesceglia che sbagliave a taglià alla cumbina, ce sariia passate propia pe ccoppa, ne me saria mangh mburtate de tutt chell che va decenn. (Angelone, 1997, p. 31)

(it. Se la capenera (è soprannome cioè 'testa con i capelli neri') non mi avesse preso tutta quella legna, per una pianticella di quercia (vescéglia) che sbagliai a tagliare ai confini, avrei proprio potuto passarci sopra, e non mi sarebbe nemmeno interessato tutto quello che andava dicendo.)

Nelle commedie dialettali di Angelone sono reperibili soltanto due esempi di quello che gli studiosi, soprattutto nord-americani, chiamano italiese (Clivio, 1986; Danesi,1986; Vizmuller-Zocco, 1995) o italoamericano (Haller, 1993), cioè quella lingua che nasce dal contatto tra l'italiano, ma più precisamente tra i dialetti parlati dagli emigrati, e l'inglese. Cenni all'uso di questa varietà sono forniti da Marcato (2012) che tratta il plurilinguismo letterario e specificamente l'opera di Capuana, Gli americani di Ràbbato, dove si riscontrano esempi ("Nuova Iorca" per 'New York' e "ferme" per 'fattorie') dell'italo-americano nelle lettere di due fratelli emigrati siciliani (cf. Marcato, 2012, p. 159). Ne La ruota della fortuna troviamo il seguente esempio di italiese pronunciato dallo zio Leonardo che torna al paese dall'America: 
16. Leonardo: Ecche nge putemme scta tiemb assia se no loch gl'ioperaie ze menane tutta la farma a zamb pe'llaria e n'avemme manghe a chi recorr. (Angelone, 1994, pp. 47-48).

(it. Leonardo: Non possiamo trattenerci qui troppo a lungo altrimenti lì gli operai mandano la fattoria all'aria e non abbiamo nemmeno nessuno a cui rivolgerci.)

Un altro esempio si trova ne La tagliola:

17. Carle: Che ppenziere tè de cchiss suold! Chiss pe mmo sceteiann buone come scteiann; chiuttosct re problema è de quire sctore che scta a re $\mathbf{N u}$ Gere. Quire è re problema. (Angelone, 2013b, p. 52)

(it. Carle: Che pensieri ha di codesti soldi? Per ora stanno bene come stanno; il problema piuttosto è di quel negozio a New Jersey. Quello è il problema!)

Gli esempi mettono in rilievo 'farma', 'sctore' e 'Nu Gere' rispettivamente dall'inglese farm, store e New Jersey. Per quanto riguarda 'sctore' si nota sia nella grafia che nella pronuncia il suono palatale della fricativa preconsonantica tipico di molti dialetti di questa zona, come notato sopra per il dimostrativo 'queshto/a'. Inoltre, questi esempi mostrano la sensibilità del commediografo che, anche non avendo mai attraversato l'oceano, evidentemente avrà sentito in actu questi termini quando gli emigrati ritornavano al paese e riesce a includere anche queste sfumature di lingua nelle sue opere per rendere il tutto più autentico.

\section{CONCLUSIONE}

Da questa breve indagine delle commedie dialettali di Antonio Angelone si nota prima di tutto l'uso dei dialetti, soprattutto quelli dei comuni della zona isernina, dialetti che vengono riprodotti fedelmente da un autore che non soltanto li conosce molto bene, ma è anche capace di metterli sulla bocca di personaggi autentici, rappresentativi del mondo popolare e contadino molisano. Si notano inoltre esempi di altre varietà sia nel repertorio dei molisani stessi che in quello degli altri protagonisti a volte o non-molisani o, se molisani, provenienti da ceti sociali elevati. Il commediografo fa alcune scelte linguistiche per rendere il tutto più credibile sul palcoscenico, per rendere più vivi e autentici i personaggi 
in generale. Si potrebbe constatare che lo fa anche per creare una distanza tra il suo popolo, che rimane fedele al patrimonio linguistico che è il dialetto nonostante qualche spia linguistica riguardante diverse forme d'italiano che i personaggi pronunciano in date situazioni, e la borghesia antica e moderna e l'esaltazione di questa fedeltà rappresenta certo il filo conduttore dell'intera opera di Angelone.

\section{BIBLIOGRAFIA}

Angelone, A. (1991). La sperimentazione dei maestri. Forli del Sannio: Proloco Forulum.

Angelone, A. (1994). La ruota della fortuna. Forli del Sannio: Edizioni "Verso il futuro".

Angelone, A. (1995). Ciccotè. New York: Edizioni il Ponte Italo-americano.

Angelone, A. (1996). Re vuasce sott'alle sctelle. New York: Edizioni il Ponte Italo-americano.

Angelone, A. (1997a). La vecchia che ne vvuleva murì. New York: Edizioni il Ponte Italo-americano.

Angelone, A. (1997b). Tra veglie e ssuonne. New York: Edizioni il Ponte Italoamericano.

Angelone, A. (2000). La banda Centrillo: Brigantaggio postunitario da Vallerotonda a Forli del Sannio. New York: Edizioni il Ponte Italo-americano.

Angelone, A. (2003). Da re semiend alla tresca. Isernia: Edizioni Accademia Internazionale Lucia Mazzocco Angelone.

Angelone, A. (2004). Re matremoneje (2nd Ed.). Isernia: Edizioni Accademia Internazionale Lucia Mazzocco Angelone.

Angelone, A. (2005). L'atrajiere, iere e vuojje. Isernia: Edizioni Accademia Internazionale Lucia Mazzocco Angelone.

Angelone, A. (2006). Felemena la lengacciuta e Alfonz re cengenare. Isernia: Edizioni Accademia Internazionale Lucia Mazzocco Angelone.

Angelone, A. (2007). Voci d'oltretomba: Da Micheleccia a Colle Pertino. Isernia: Edizione Accademia Internazionale Lucia Mazzocco Angelone.

Angelone, A. (2013a). Fuga in paradiso. Isernia: EdiAccademia.

Angelone, A. (2013b). La tagliola. Isernia: EdiAccademia.

Angelone, A. (2015). Glossario dialettale. Isernia: EdiAccademia. 
Avolio, F. (2000). Prefazione. In A. Angelone. La banda Centrillo: Brigantaggio postunitario da Vallerotonda a Forli del Sannio (pp. 7-10). New York: Edizioni il Ponte Italo-americano.

Avolio, F. (2002). Il Molise. In M. Cortelazzo, C. Marcato, N. De Blasi \& G.P. Clivio (Eds.), I dialetti italiani: storia, struttura e uso (pp. 604627). Torino: UTET.

Avolio, F. (2005). Prefazione. In A. Angelone, L'atrajiere, iere e vuojje (pp. 11-16). Isernia: Edizioni Accademia Internazionale Lucia Mazzocco Angelone.

Bagnoli, N.L., Discenza, M., \& Faralli, G.B. (2001). Dizionario dialettale. Lessico comparato dei comuni molisani compresi nelle valli interne del Biferno, del Tigno e del Volturno. Venafro: Edizioni Vitmar.

Barberi Squarotti, G. (2009). Comunicazione personale a Antonio Angelone. In Antologia critica. Commedie e poesie di Antonio Angelone (p. 187). L'Aquila: Pubbliemme.

Beccaria, G.L. (1975). Letteratura e dialetto. Bologna: Zanichelli.

Berruto, G. (1987). Sociolinguistica dell'italiano contemporaneo. Roma: La Nuova Italia Scientifica.

Carlson, M. (2006). Speaking in Tongues: Language at Play in the Theatre. Ann Arbor: University of Michigan Press.

Cecchi, G. (2003). Il pregio della commedia dialettale di Antonio Angelone. In AAVV., Commedie e poesie di Antonio Angelone: Antologia Critica (pp. 41-45). Isernia: Edizioni Accademia Internazionale Lucia Mazzocco Angelone.

Clivio, G.P. (1986). Competing Loanwords and Loanshifts in Toronto's italiese. In C. Bettoni (Ed.), Altro Polo: Italian Abroad. Studies on Language Contact in English-Speaking Countries (pp. 129-146). Sydney: University of Sydney.

Clivio, G.P. (2005). Postfazione. In A. Angelone, L'atrajiere, iere e vuojje (pp. 65-68). Isernia: Edizioni Accademia Internazionale Lucia Mazzocco Angelone.

Colabella, M. (1993). Dizionario illustrato bonefrano-italiano. Bonefro: Università della terra di Venifro.

Cortelazzo, M. (1972). Lineamenti di italiano popolare. Pisa: Pacini.

Croce, B. (1927). La letteratura dialettale riflessa, la sua origine nel Seicento e il suo ufficio storico. In B. Croce, Uomini e cose della vecchia Italia (pp. 225-234). Bari: Laterza, 
D’Achille, P. (1994). L'italiano dei semicolti. In L. Serianni \& P. Trifone (Eds.), Storia della lingua italiana (pp. 41-79). Torino: Einaudi.

Danesi, M. (1986). Canadian Italian: A Case in Point of How Language Adapts to Environment. Polyphony, 7, 111-114.

De Martino Cappuccio, A. (2012). The relationship between dialect, theatre and power in Antonio Gramsci. The Italianist, 32, 67-38.

De Mauro, T. (1970). Per lo studio dell'italiano popolare unitario. In A. Rossi (Ed.), Lettere da una tarantata (pp. 43-75). Bari: De Donato.

Folena, G. (1983). Le lingue della commedia è la commedia delle lingue. In P. Benincà (Ed.), Scritti linguistici in onore di Giovan Battista Pellegrini (pp. 1485-1513). Pisa: Pacini.

Folena, G. (1991). Il linguaggio del caos. Studi sul plurilinguismo rinascimentale. Torino: Bollati Boringhieri.

Haller, H.W. (1999). The Other Italy: The Literary Canon in Dialect. Toronto: University of Toronto Press.

Haller, H.W. (1993). Una lingua perduta e ritrovata. L'italiano degli italoamericani. Firenze: La Nuova Italia.

Haller, H.W. (2004). Innovative Transitions: Exploring Italy's Literary Dialects. In D. Brancato, K. Reynolds, P. Chirumbolo \& F. Calabrese (Eds.), Transitions: Prospettive di studio sulle trasformazioni letterarie e linguistiche nella cultura italiana (pp. 3-15). Fiesole: Cadmo.

Iannacito-Provenzano, R. (2006). Il dialetto molisano di Villa San Michele (IS): Fonologia, morfologia, sintassi e lessico. Ottawa: Legas.

Iannacito-Provenzano, R. (2009). L'etnografia nelle commedie dialettali di Antonio Angelone. In R. Longo-Lavorato (Ed.), De vulgari eloquentia: lingua e dialetti nella cultura italiana (pp. 89-102). Ottawa: Legas.

Iannacito, R. (2005). Cenni sulla frase ipotetica in due dialetti dell'Alto Molise. Forum Italicum 39(2), 498-519.

Iemma, S. (1997). La commedia Ciccotè di Antonio Angelone vista come un fatto culturale. In AAVV., Commedie e poesie di Antonio Angelone (pp. 81-83). New York: Edizioni il Ponte Italo-americano.

Jaberg, K., \& Jud, J. (1932). Sprach -und Sachatlas Italiens und der Südschweiz. Zofingen: Verlagsantalt Ringier \& Co.

Marcato, C. (2012). Il plurilinguismo. Bari: Laterza.

Martelli, S. (1996a). La letteratura dialettale molisana. In AAVV., Lingua e dialetto nella tradizione letteraria italiana. Atti del Convegno di Salerno 5-6 novembre (pp. 273-328). Roma: Salerno Editrice. 
Martelli, S. (1996b). Prefazione. In A. Angelone, Re vuasce sott'alle sctelle (pp. 7-10). New York: Edizioni il Ponte Italo-americano.

Minadeo, M. (1996). Lessico del dialetto di Ripalimosani. Campobasso: Edizioni Enne.

Nencioni, G. (1983). Di scritto e di parlato. Discorsi linguistici. Bologna: Zanichelli.

Paccagnella, I. (1984). Il fasto delle lingue. Plurilinguismo letterario nel Cinquecento. Roma: Bulzoni.

Pacitti, E. (2014). Un autore alla ricerca del vero senso dell'essere. In AAVV., Antonio Angelone alla ribalta (pp. 14-19). Isernia: EdiAccademia.

Paone, N. (2006). Introduzione. In A. Angelone, Felemena la lengacciuta e Alfonz re cengenare (pp. 11-13). Isernia: Edizioni Accademia Internazionale Lucia Mazzocco Angelone.

Piemontese, P. (1993). L'A tonica nei dialetti molisani. Foggia: Ennio Cappetta.

Rohlfs, G. (1966). Grammatica storica della lingua italiana e dei suoi dialetti. $I \&$ II. Torino: Einaudi.

Sabatini, F. (1985). L'“italiano dell'uso medio": una realtà tra le varietà linguistiche italiane. In G. Holtus \& E. Radke (Eds.), Gesprochenes Italienisch in Geschichte und Gegenwart (pp. 154-185). Tübingen: Narr.

Santilli, C. (1988). Isernia e il suo dialetto. Volume I. Isernia: E. Di. Ci.

Serianni, L., \& Trifone, P. (Eds.). (1994). Storia della lingua italiana. Torino: Einaudi.

Trifone, P. (2000). L'italiano a teatro. Dalla commedia rinascimentale a Dario Fo. Roma: Istituti Editoriali e Poligrafici Internazionali.

Troiano, R. (2013). Prefazione. In A. Angelone, La tagliola (pp. 5-8). Isernia: EdiAccademia.

Vizmuller-Zocco, J. (1995). The Languages of Italian Canadians. Italica, 72 (4), 512-529.

Riassunto: Quest'articolo tratta il plurilinguismo nelle commedie dialettali del molisano Antonio Angelone. Dopo alcuni riferimenti biografici su Angelone, viene commentato, in prima luogo, il dialetto ricco, espressivo e autentico posto sulla bocca di personaggi spesso tratti dal mondo contadino ben noto all'autore. Inoltre sono forniti dettagli sulla presa di coscienza, da parte del commediografo, delle sfide riguardanti lo scrivere in dialetto. La parte fondamentale di questo saggio verte comunque sugli aspetti plurilinguistici che, non certamente estranei alla produzione letteraria in Italia, si manifestano pienamente nelle commedie angeloniane attraverso personaggi creati da un 
autore che riesce a mettere in rilievo come sia importante impiegare varietà linguistiche adatte alle circostanze e alle esigenze dei protagonisti per rendere il teatro più autentico, più credibile per il lettore/lo spettatore. Insieme al dialetto o ai dialetti, nel repertorio dei protagonisti si riscontrano esempi della lingua italiana, dell'italiano popolare e dell'italiese ovvero dell'italo-americano, pronunciati da personaggi che appartengono a diverse generazioni, a diverse classi sociali e anche che provengono da diversi comuni, regioni o paesi.

Keywords: Plurilinguismo letterario; commedie dialettali; dialetto; italiano popolare; molisano 